\title{
Endoscopic and surgical ampullectomy for non-invasive ampullary tumors: Short-term outcomes
}

\author{
Margaux Dubois ${ }^{1,}$, Ismail Labgaa ${ }^{1, \S}$, Gian Dorta $^{2, *}$, Nermin Halkic ${ }^{1}$ \\ ${ }^{1}$ Department of Visceral Surgery, Lausanne University Hospital (CHUV), Lausanne, Switzerland; \\ ${ }^{2}$ Department of Gastroenterology and Hepatology, Lausanne University Hospital (CHUV), Lausanne, Switzerland.
}

\begin{abstract}
Summary Non-invasive ampullary tumors, may be treated with endoscopic (EA) or surgical ampullectomy (SA). However, evidence on the morbidity of these techniques remains limited. This pilot study aimed to assess and compare morbidity of EA and SA. Patients undergoing EA or SA for non-invasive ampullary tumors were retrospectively analyzed and compared. Outcomes were postoperative complications graded with Clavien Classification and Comprehensive Complication Index (CCI), and length of stay (LoS). A review of the literature was performed to propose an evidence-based algorithm to treat ampullary tumors. A total of 11 EA and 19 SA were identified and analyzed. EA was associated with shorter intervention (51 vs. $191 \mathrm{~min}, p<0.001)$ and decreased blood loss $(0$ vs. $100 \mathrm{~mL}, p<0.001)$. Postoperative complications were more frequent after surgery compared to endoscopy $(9 \% v s .68 \%, p$ $=0.002)$. Surgical patients showed a higher CCI $(0$ vs. 8.7, $p<0.001)$. LoS was reduced in patients undergoing endoscopy ( 0 vs. 14 days, $p<0.001)$, with comparable readmissions rates $(p=0.126)$. Necessity of subsequent treatment was more frequent after endoscopic, compared to SA (5 vs. 1, $p=0.016)$. EA was associated with lower morbidity than SA and appeared as an appropriate first-line treatment for non-invasive ampullary tumors. SA remains a valuable alternative after EA failure.
\end{abstract}

Keywords: Ampulloma, ampullectomy, endoscopy, postoperative complications, morbidity

\section{Introduction}

Ampullary neoplasms are rare tumors accounting for $0.5 \%$ of gastrointestinal tumors, and displaying distinctive features related to their anatomical and pathological singularities (1). There is strong evidence for the risk of benign lesion to transform into malignant carcinoma (2). As a consequence, the indication to resect ampullary neoplasms, regardless of their grade, is widely accepted (1). Strikingly, no dedicated guidelines for the treatment of these tumors have been proposed so far. Although pancreaticoduodenectomy (PD) has been evidenced as the treatment of choice for invasive ampullary tumors $(1,3)$, its high morbidity and

Released online in J-STAGE as advance publication December $18,2016$.

${ }^{\S}$ These authors contributed equally to this works.

*Address correspondence to:

Dr. Gian Dorta, Department of Gastroenterology and Hepatology, University Hospital of Lausanne (CHUV), CH-1011 Lausanne, Switzerland.

E-mail: Gian.Dorta@chuv.ch mortality may not be justified for non-invasive tumors. (4-6). Indeed, less invasive techniques such as surgical ampullectomy (SA) and endoscopic ampullectomy (EA) have been acknowledged as appropriate approaches for this indication (7-11). While a few studies comparing SA vs. EA have been reported, the morbidity related to these techniques has not been thoroughly assessed and compared $(7,12)$.

This study thus aimed to provide a comprehensive assessment and comparison of the morbidity induced by EA and SA. In addition, it sought to generate a decision-making algorithm to treat ampullary neoplasms, primarily based on the available evidence from the literature.

\section{Materials and Methods}

This retrospective cohort study was conducted in the Departments of Visceral Surgery and Gastroenterology \& Hepatology, Lausanne University Hospital (CHUV). This study was conducted in accordance with the STROBE criteria (http://strobe-statement.org/) and 
registered under www.researchregistry.com (UIN: 577).

\subsection{Patients and procedures}

Patients undergoing EA or SA over the last 10 years (from 2005 to 2015) were identified from our prospective databases. Age $<18$ years, immunosuppressive treatment and emergency surgery were considered as exclusion criteria. EA and SA were performed according to techniques described previously $(7,12)$. Non-invasive ampullary tumor was defined as adenoma or in situ adenocarcinoma.

\subsection{Data collection}

Relevant demographics, comorbidities, preoperative assessment, details of the procedure and clinical outcomes were prospectively collected and anonymized in a computerized database. Operation duration was measured from incision to skin closure by the anesthetists. Intraoperative blood loss was estimated measuring the volume of aspirated fluid and soaked gauzes, jointly by the anesthetists and the surgeons. Postoperative complications were assessed by both the Clavien Classification (13) and the Comprehensive Complication Index (CCI) (14) within 30 postoperative days. Briefly, CCI is a score - ranging from 0 (no complication) to 100 (death) - that sums each postoperative complication. By avoiding underreported minor complications, $\mathrm{CCI}$ is a robust metric to assess morbidity. Length of stay (LoS) was calculated from day of surgery until discharge. Readmissions were considered within 30 postoperative days.

Electronic search was performed using MEDLINE/ PubMed, Embase, Web of Knowledge, and The Cochrane Library. The search strategy was applied between January 1990 and June $30^{\text {th }}$ 2016. Terms used were ampullectomy, ampulloma, ampullary tumor. Data from this systematic search were used to generate an algorithm for decision-making (Figure 1).

\subsection{Statistical analysis}

Normal continuous and categorical variables were compared using Student's $t$ test and Chi-squared, respectively. A $p$ value $<0.05$ was considered to be statistically significant. Data analyses were performed using SPSS v20 statistical software (Chicago, IL).

\section{Results and Discussion}

\subsection{Patients demographics and tumors characteristics}

A total of 30 ampullectomies - with 11 EA and 19 SA - involving 24 patients were identified and analyzed. In term of demographics, the 2 groups were comparable for most variables (Table 1). On average, surgical patients were 4 years older than endoscopic patients (65 vs. 69 years, $p=0.024$ ). None of the other demographics and comorbidities showed significant difference between the groups.

Tumors also displayed comparable characteristics in the 2 groups, with no difference in term of histological type $(p=0.573)$ or size $(p=0.953)$ (Table 1$)$.

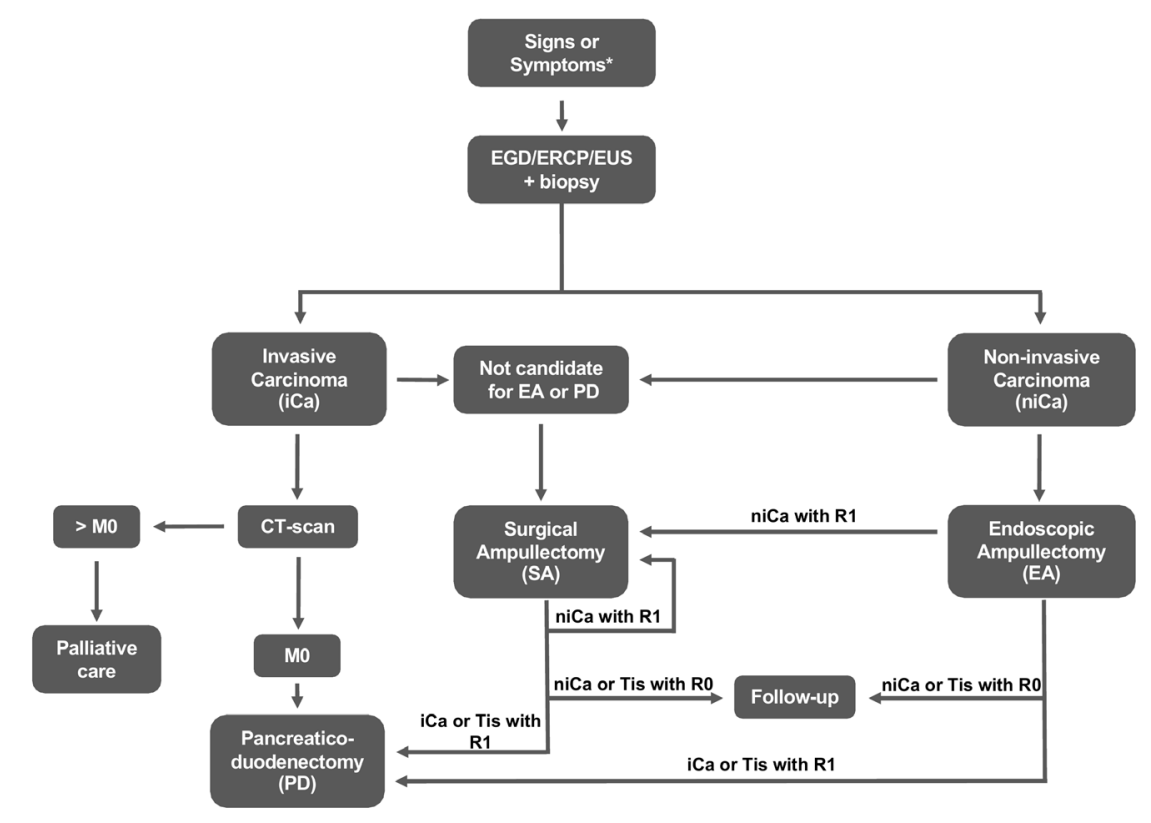

Figure 1. Decision-making of the treatment of ampullary tumors: an algorithm based on the evidence in the literature. EGD: Esophagogastroduodenoscopy. ERCP: Endoscopic Retrograde Cholangio-pancreatography. EUS: Endoscopic Ultrasound. niCa: Non-invasive Carcinoma. iCa: Invasive Carcinoma. Tis: Adenocarcinoma in situ. R0: Negative resection margins. R1: Positive resection margins. M0: Absence of distant metastasis. CT-scan: Computed Tomography. EA: Endoscopic Ampullectomy. SA: Surgical Ampullectomy. PD: Pancreaticoduodenectomy. 
Table 1. Characteristics of patients and tumors

\begin{tabular}{|c|c|c|c|}
\hline Items & Endoscopic ampullectomy $(n=11)$ & Surgical ampullectomy $(n=19)$ & $p$-value \\
\hline Median age (years) & $65(52-74)$ & $69(67-81)$ & 0.024 \\
\hline Gender (female) & $7(64)$ & $12(63)$ & 1.000 \\
\hline ASA I/II & $8(80)$ & $14(74)$ & 1.000 \\
\hline Median BMI $\left(\mathrm{kg} / \mathrm{m}^{2}\right)$ & $25(21-27)$ & $25(21-28)$ & 0.667 \\
\hline Diabetes & 0 & $1(5.3)$ & 1.000 \\
\hline Smoker & $3(27)$ & $7(37)$ & 0.702 \\
\hline Severe heart disease & $3(27)$ & $6(32)$ & 1.000 \\
\hline Severe pulmonary disease & $1(9)$ & $3(16)$ & 1.000 \\
\hline Immunosuppression & 0 & 0 & \\
\hline FAP & $3(27)$ & $1(5.3)$ & 0.126 \\
\hline Type of tumor & & & 0.573 \\
\hline Inflammatory & $1(10)$ & $2(11)$ & \\
\hline Adenoma & $8(80)$ & $12(63)$ & \\
\hline Adenocarcinoma (Tis) & $1(10)$ & $5(26)$ & \\
\hline Median Tumor size (mm) & $15(9-20)$ & $14(10-19)$ & 0.953 \\
\hline
\end{tabular}

EA: Endoscopic Ampullectomy. BMI: Body Mass Index. SA: Surgical Ampullectomy. FAP: Familial Adenomatous Polyposis. Tis: Adenocarcinoma in situ.

Table 2. Outcomes

\begin{tabular}{|c|c|c|c|}
\hline Items & Endoscopic ampullectomy $(n=11)$ & Surgical ampullectomy $(n=19)$ & $p$-value \\
\hline Median duration (min) & $51(29-71)$ & $191(181-210)$ & $<0.001$ \\
\hline Median blood loss (mL) & 0 & $100(0-200)$ & $<0.001$ \\
\hline \multicolumn{4}{|l|}{ Postoperative complications } \\
\hline Overall & $1(9)$ & $13(68)$ & 0.002 \\
\hline Minor I-II & $1(9)$ & $10(53)$ & 0.023 \\
\hline Major III-IV & 0 & $6(32)$ & 0.061 \\
\hline Grade V & 0 & 0 & - \\
\hline CCI & 0 & $9(0-34)$ & $<0.001$ \\
\hline Median length of stay (days) & 0 & $14(10-30)$ & $<0.001$ \\
\hline Readmission & $3(27)$ & $1(5)$ & 0.126 \\
\hline Further treatment requested & $5(45)$ & $1(5)$ & 0.016 \\
\hline
\end{tabular}

EA: Endoscopic Ampullectomy. SA: Surgical Ampullectomy. CCI: Comprehensive Complication Index.

\subsection{Preoperative assessment}

All of the patients underwent an endoscopic examination with either an Esophagogastroduodenoscopy (EGD), Endoscopic Retrograde Cholangio-pancreatography (ERCP) or Endoscopic Ultrasound (EUS) with subsequent biopsies. No further assessment is recommended for patients without invasive cancer and macroscopic features of malignancy (6). Patients who do not meet these two criteria should undergo a Computed Tomography scan (CT-scan) to exclude a metastasize disease, which will indicate the necessity of a palliative care.

Tumor size was assessed either by CT-scan or EUS. A cut-off of $2 \mathrm{~cm}$ was used to allocate treatment, namely EA or SA.

\subsection{Outcomes}

Intra- and postoperative outcomes are summarized in Table 2. Endoscopy was associated with shorter intervention (51 vs. $191 \mathrm{~min}, p<0.001)$ and decreased blood loss (0vs.100 mL, $p<0.001)$. Overall, surgical patients experienced more postoperative complications
( $9 \%$ vs. $68 \%, p=0.002)$. Minor complications were significantly increased in the surgery group ( $9 \%$ vs. 53\%, $p=0.023)$, whereas a trend was observed for major complications ( $0 \%$ vs. 32\%, $p=0.061)$. Consistently, surgical patients showed a higher median CCI (0 vs. $8.7, p<0.001)$. LoS was reduced in patients undergoing EA (0 vs. 14 days, $p<0.001)$, without increasing the readmissions $(27 \%$ vs. $5 \%, p=0.126)$. The proportion of patients necessitating subsequent treatment was higher in EA compared to SA (5 vs. 1, $p=0.016$ ). Four patients initially treated with EA had to undergo SA for technical resectability reasons, while 1 patient underwent 2 EA procedures before necessitating SA; of note, the patient had familial adenomatous polyposis (FAP). In the SA group, 1 patient subsequently underwent PD because of recurrence.

\subsection{Conclusions}

EA was associated with more favorable outcomes than $\mathrm{SA}$, in this cohort of patients treated for non-invasive ampullary tumors. Patients undergoing EA indeed showed lower complications rate and reduced LoS, in comparison to patients treated with SA. 
Comparative studies on these less invasive alternatives reported that EA was associated with lower morbidity compared to SA $(7,12)$. The comparison with previous studies is somehow awkward since they did not focus on postoperative complications, but rather reported overall morbidity without using a validated grading system $(7,12)$. Notwithstanding, a landmark study by Ceppa et al. showed an overall morbidity of $18 \%$ for EA compared to $42 \%$ for patients undergoing surgery $(p=0.006)(7)$; these findings are indeed consistent with the present results.

Some drawbacks of the study need to be addressed. The study is mainly limited by its retrospective design and the small sample size, which may potentially influence the findings. Although it was not the aim of this study, assessing prognosis of patients undergoing EA vs. SA would enable to decipher whether one approach could offer longer survival than the other. There is an imperative need to design multicentric prospective studies in patients with ampullary tumors, to answer questions such as long-term survival, in this field. Nevertheless, the present study precisely detailed the landscape of complications after EA and SA. One could argue that the 2 groups were different for age; although significant, this difference was minor (65 vs. 69 years, $p=0.024$ ) and is thus unlikely to be the only cause of the difference observed in outcomes. This report may serve as a tool to preoperatively provide precise risk rates to the patients.

Strikingly, there is a clear unmet need to define a clear consensus for the treatment of ampullary tumors. With this in mind, Figure 1 aimed to propose an algorithm to guide the decision-making for the preoperative assessment and for the treatment of ampullary neoplasms, primarily based on the evidence from the literature $(3,7,8,12,15)$. This algorithm may be particularly pertinent to help gastroenterologists and hepatopancreatobiliary surgeons, to tailor their decision-making for the treatment of patients with noninvasive ampullary tumors. Patients frequently present with obstructive jaundice ( $20 \%$ in this cohort), but a variety of other unspecific symptoms may also occur, such as: abdominal pain, fatigue, weight loss or acute pancreatitis. The first step is to exclude gallstones, typically by abdominal ultrasound (US). The following measure is to perform tissue biopsies, which is critical for diagnostic purpose. This may be achieved either by ERCP/EGD or by EUS. The latter demonstrated a high sensitivity and is particularly accurate for the T staging which is key element since it determines the therapeutic modality (1,16-18). Further investigation with an abdominal CT-scan, should be performed if the preoperative biopsies reveal an invasive carcinoma and/or if macroscopic malignant characteristics are observed during EGD/ERCP/EUS. These findings include features such as friable, ulcerative or hemorrhagic lesion, Oddi's sphincter invasion and extension to common bile duct or pancreatic duct $(1,7,15)$. In case of systemic disease, palliative care such as stenting could relieve patient's symptoms. The presence of invasive patterns is an indication for $\mathrm{PD}$, unless the patient has contraindication for major surgery, in which case SA appears as a reasonable option (17). In case of non-invasive tumor, EA would be first considered given its lower morbidity, as confirmed by the present results. Nonetheless, SA is an alternative after EA failure or if EA is not amenable $(8,15,18,19)$. The EA failure rate is indeed substantial, both in this series and in the literature. In this context, SA offers an appealing alternative that is efficient and safer than PD (19). If remaining malignancy is evidenced by the pathological analysis after either EA or SA, PD is further recommended. Conversely, if no evidence of malignancy is found, a follow-up with endoscopic surveillance is appropriate.

In summary, the present results suggest that EA may be associated with lower morbidity than SA, and may be considered as a first-line treatment for patients with non-invasive ampullary tumors. SA remains however a useful option after EA failure, or in patients with contraindications for EA or PD.

\section{References}

1. Panzeri F, Crippa S, Castelli P, Aleotti F, Pucci A, Partelli S, Zamboni G, Falconi M. Management of ampullary neoplasms: A tailored approach between endoscopy and surgery. World J Gastroenterol. 2015; 21:7970-7987.

2. Seifert E, Schulte F, Stolte M. Adenoma and carcinoma of the duodenum and papilla of Vater: A clinicopathologic study. Am J Gastroenterol. 1992; 87:37-42.

3. Askew J, Connor S. Review of the investigation and surgical management of resectable ampullary adenocarcinoma. HPB (Oxford). 2013; 15:829-838.

4. Menahem B, Guittet L, Mulliri A, Alves A, Lubrano J. Pancreaticogastrostomy is superior to pancreaticojejunostomy for prevention of pancreatic fistula after pancreaticoduodenectomy: An updated metaanalysis of randomized controlled trials. Ann Surg. 2015; 261:882-887.

5. Mathur A, Paul H, Ross S, Luberice K, Hernandez J, Vice M, Rosemurgy AS. Transduodenal ampullectomy for ampullary adenomas: A safe and effective procedure with long-term salutary outcomes. Am Surg. 2014; 80:185-190.

6. Gao Y, Zhu Y, Huang X, Wang H, Huang X, Yuan Z. Transduodenal ampullectomy provides a less invasive technique to cure early ampullary cancer. BMC Surg. 2016; 16:36.

7. Ceppa EP, Burbridge RA, Rialon KL, Omotosho PA, Emick D, Jowell PS, Branch MS, Pappas TN. Endoscopic versus surgical ampullectomy: An algorithm to treat disease of the ampulla of Vater. Ann Surg. 2013; 257:315-322.

8. Salmi S, Ezzedine S, Vitton V, Menard C, Gonzales JM, Desjeux A, Grimaud JC, Barthet M. Can papillary carcinomas be treated by endoscopic ampullectomy? Surg Endosc. 2012; 26:920-925. 
9. Branum GD, Pappas TN, Meyers WC. The management of tumors of the ampulla of Vater by local resection. Ann Surg. 1996; 224:621-627.

10. de Castro SM, van Heek NT, Kuhlmann KF, Busch OR, Offerhaus GJ, van Gulik TM, Obertop H, Gouma DJ. Surgical management of neoplasms of the ampulla of Vater: Local resection or pancreatoduodenectomy and prognostic factors for survival. Surgery. 2004; 136:9941002.

11. Clary BM, Tyler DS, Dematos P, Gottfried M, Pappas $\mathrm{TN}$. Local ampullary resection with careful intraoperative frozen section evaluation for presumed benign ampullary neoplasms. Surgery. 2000; 127:628-633.

12. Onkendi EO, Naik ND, Rosedahl JK, Harmsen SW, Gostout CJ, Baron TH, Sr., Sarr MG, Que FG. Adenomas of the ampulla of Vater: A comparison of outcomes of operative and endoscopic resections. J Gastrointest Surg. 2014; 18:1588-1596.

13. Dindo D, Demartines N, Clavien PA. Classification of surgical complications: A new proposal with evaluation in a cohort of 6336 patients and results of a survey. Ann Surg. 2004; 240:205-213.

14. Slankamenac K, Graf R, Barkun J, Puhan MA, Clavien PA. The comprehensive complication index: A novel continuous scale to measure surgical morbidity. Ann Surg. 2013; 258:1-7.
15. Laleman W, Verreth A, Topal B, Aerts R, Komuta M, Roskams T, Van der Merwe S, Cassiman D, Nevens F, Verslype C, Van Steenbergen W. Endoscopic resection of ampullary lesions: A single-center 8-year retrospective cohort study of 91 patients with long-term follow-up. Surg Endosc. 2013; 27:3865-3876.

16. Skordilis P, Mouzas IA, Dimoulios PD, Alexandrakis G, Moschandrea J, Kouroumalis E. Is endosonography an effective method for detection and local staging of the ampullary carcinoma? A prospective study. BMC Surg. 2002; 2:1.

17. Song J, Liu H, Li Z, Yang C, Sun Y, Wang C. Longterm prognosis of surgical treatment for early ampullary cancers and implications for local ampullectomy. BMC Surg. 2015; 15:32.

18. Ardengh JC, Kemp R, Lima-Filho ER, Dos Santos JS. Endoscopic papillectomy: The limits of the indication, technique and results. World J Gastrointest Endosc. 2015; 7:987-994.

19. Schneider L, Contin P, Fritz S, Strobel O, Buchler MW, Hackert T. Surgical ampullectomy: An underestimated operation in the era of endoscopy. HPB (Oxford). 2016; 18:65-71.

(Received October 19, 2016; Revised November 16, 2016; Accepted November 29, 2016) 